\title{
Non-genetic inheritance of environmental exposures: a protocol for a map of systematic reviews with bibliometric analysis
}



\begin{abstract}
Background: Over the last few decades, we increasingly see examples of parental environmental experiences influencing offspring health and fitness. More recently, it has become clear that some non-genetic effects can be conferred across multiple generations. This topic has attracted research from a diversity of disciplines such as toxicology, biomedical sciences, and ecology, due to its importance for environmental and health issues, as well as ecological and evolutionary processes, with implications for environmental policies. The rapid accumulation of primary research has enabled researchers to perform systematic reviews (SRs), including meta-analyses, to investigate the generality of and sources of variation in non-genetic effects. However, different disciplines ask different questions and SRs can vary substantially in scope, quality, and terminology usage. This diversity in SRs makes it difficult to assess broad patterns of non-genetic effects across disciplines as well as determine common areas of interest and gaps in the literature. To clarify research patterns within the SR literature on non-genetic inheritance, we plan to create a map of systematic reviews as well as conduct bibliometric mapping (referred to as'research weaving'). We will address four key questions: first, what are the broad research patterns unifying the SR literature on non-genetic inheritance across disciplines? Second, are there discipline-specific research patterns, including terminology use, between disciplines? Third, how are authors of the SR literature connected? Fourth, what is the reliability of the SR literature?

Methods: We will systematically collect reviews within the SR 'family' that examine non-genetic inheritance arising from parental and ancestral environment by searching databases for journal articles and grey literature, as well as conducting backwards and forwards searching. Search hits will be double screened using 'decision trees' that represent the inclusion criteria. All relevant data elements on the review's topic, as well as a critical appraisal of the review's approach and reporting, will be extracted into Excel flat sheets. Bibliometric data will be directly extracted from Scopus. We will then query all relevant data elements to address our objectives and present outcomes in easily interpretable tables and figures, accompanied by a narrative description of results.
\end{abstract}

Keywords: Environmental effects, Scoping review, Inter-generational inheritance, Trans-generational inheritance, Maternal effects, Paternal effects

*Correspondence: e.macartney@unsw.edu.au

†Shinichi Nakagawa and Malgorzata Lagisz contributed equally as senior authors

Evolution and Ecology Research Centre, School of Biological, Earth and Environmental Sciences, University of New South Wales, Sydney, NSW, Australia

\begin{abstract}
Background
Non-genetic inheritance associated with differences in parental environment is now known to be widespread and diverse: from the impacts of the "Dutch Hunger Winter" on offspring metabolism and health [1] to the effects of climate on offspring physiology and behaviour [2]. Many non-genetic effects are due to maternal provisioning to
\end{abstract} original author(s) and the source, provide a link to the Creative Commons licence, and indicate if changes were made. The images or other third party material in this article are included in the article's Creative Commons licence, unless indicated otherwise in a credit line to the material. If material is not included in the article's Creative Commons licence and your intended use is not permitted by statutory regulation or exceeds the permitted use, you will need to obtain permission directly from the copyright holder. To view a copy of this licence, visit http://creativecommons.org/licenses/by/4.0/. The Creative Commons Public Domain Dedication waiver (http://creativeco mmons.org/publicdomain/zero/1.0/) applies to the data made available in this article, unless otherwise stated in a credit line to the data. 
the developing embryo [3], but both maternal and paternal environment can induce changes in epigenetic factors associated with the germline, altering gene-expression in the following generation [4-6]. Such 'direct' effects of parental environment on the developing embryo and germline are known as 'inter-generational effects' (Fig. 1). Moreover, recent evidence has shown that non-genetic inheritance can occur beyond the generation exposed to parental condition as an embryo or germ-cell ('trans-generational inheritance'; see Fig. 1) [7-9]. This suggests that at least some environment-induced changes can be maintained across multiple generations due to the persistence of non-genetic information [10-12]. The combination of 'inter-generational' and 'trans-generational' inheritance are broadly referred to as 'non-genetic inheritance' (note, that there are also other terms often used to describe these phenomena [13]).

The rapid accumulation of primary research in nongenetic inheritance has enabled disciplines, from biomedicine and toxicology to ecology and evolution, to make

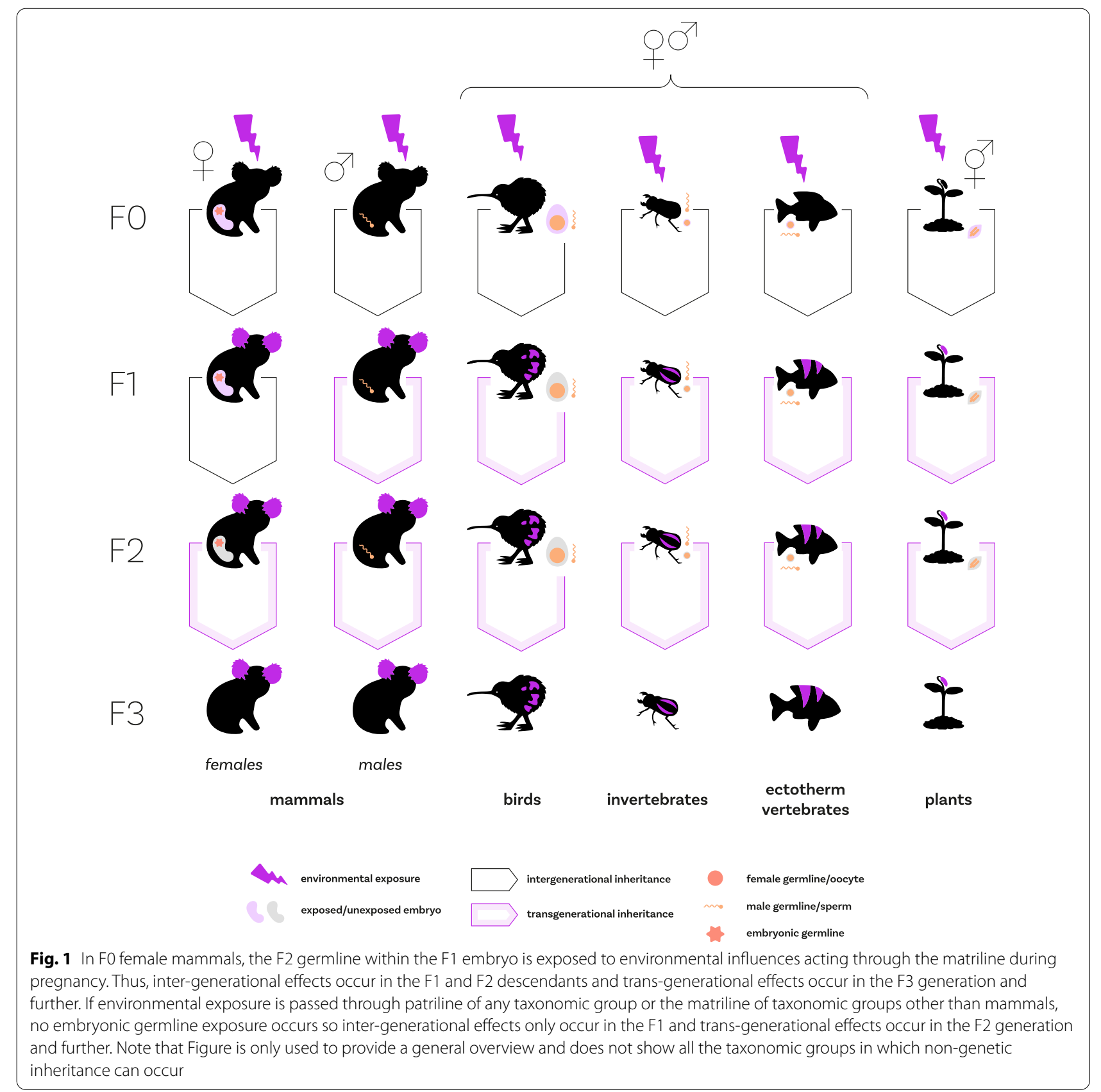


advances in our understanding of inherited diseases and syndromes [14], the effects of climate change and environmental pollutants on individual and population health $[2,15]$, as well as make substantial developments in ecological and evolutionary theory [16]. The growth of primary literature has also enabled researchers to transition into secondary research in the form of systematic reviews (hereafter "SRs"), including quantitative reviews such as meta-analyses. SRs generally follow a clear and structured framework that allows researchers to assess the consistency of effects across studies, the ubiquity of patterns across diverse study systems, as well as determine factors that contribute to the variation in such effects. However, due to the often-disparate questions and scope of different research disciplines, this has resulted in a large diversity of SRs examining patterns of non-genetic inheritance. For example, toxicological and biomedical SRs might ask if specific environmental stressors and pollutants have negative consequences for specific offspring traits, and these syntheses may focus on a taxonomically narrow group of species (e.g., common biomedical study systems such as rodents) [17-19]. In contrast, ecologists may synthesise studies across a wide diversity of environments, traits, taxa, and life stages [20,21], which is important in determining sources of variation and can help make decisions for conservation and management. Such questions are important for individual disciplines, but diverse synthesis scopes can make it difficult to determine more overarching patterns between disciplines.

In addition to differences in the scope of SRs, they can vary in terminology. For example, there are many terms used in the field of non-genetic inheritance research, such as 'inter-generational inheritance', 'trans-generational inheritance,' 'maternal effects,' 'paternal effects', 'parental effects', and 'epigenetic inheritance'. In some disciplines, these terms have very specific meanings, but they are also used interchangeably in others. Thus, there is a need to highlight patterns of terminology use to make identification of relevant literature across disciplines easier and to improve cross-disciplinary literacy [13].

SRs are generally assumed to be the most rigorous form of summarising primary literature due to the common use of conducting and reporting guidelines [20]. However, SRs can be as diverse as primary studies, varying in review design and methods, or breadth and depth [21]. They can be seen as a 'family of reviews' [22] sharing a common aim of summarising the research literature, ideally using explicit and reproducible methods. Some members of this family, like classical SRs, are focused and prescriptive in their approach, while others, like scoping reviews and systematic maps, are interested in broad patterns of evidence, terminology or even methodology of primary studies.
Naturally, it is possible to conduct a review of SRs. The purpose of such an exercise would be similar: to bring together existing knowledge, look for patterns, gaps and even rigour in existing reviews. A suitable form of synthesising 'reviews' could be a 'map' [22] (i.e., a 'map of systematic reviews'; note that the use of review maps is common in the environmental sciences, but this approach is closely related to 'scoping reviews' which are more common in the medical sciences [23]). Each SR in a map can be subjected to critical appraisal with a relevant assessment tool (e.g., Collaboration for Environmental Evidence Synthesis Assessment Tool, CEESAT [24]) to evaluate methodological transparency and the robustness of conclusions.

Mapping the secondary literature, including its quality, is integral to advancing multiple research fields-including environmental toxicology, climate change, health, agriculture, and ecology and evolution-by not only progressing scientific knowledge and identifying gaps in the literature, but improving transparency and reproducibility. Such research will also be of benefit to policymakers due to the persistence of environmental effects across generations, with implications for not only human health and fitness but that of animals and plants, including threatened species and those important for agriculture and fisheries. Furthermore, showing research 'connections' through co-author and collaboration networks, as well as how citations are connected, will enable identification of research hubs and the degree of collaboration within non-genetic inheritance research. Therefore, we propose to conduct a map of systematic reviews, including a critical appraisal, with bibliometric analysiscoined 'research weaving' [25]. This method is novel by not only providing an overview of the current state of the literature, but also showing how evidence is connected.

\section{Stakeholder engagement}

The stakeholders in this project are scientists as well as policy makers from across disciplines that are broadly interested in environmental effects on descendants. Specific stakeholders were not involved in the planning or question formulation of this project.

\section{Objectives}

For the first time, we will combine a map of systematic reviews (i.e., a scoping review of secondary literature from the SR family) with a bibliometric analysis, to deliver insights on the state of research on non-genetic inheritance of environmental exposures from across all relevant domains of research. Specifically, we will examine the literature that uses the systematic review (or related) approach to summarise the effects of F0 
(ancestral) environmental exposures on descendants (F1 and beyond) in non-human animals and plants (also see "Article screening and study eligibility criteria" section for the description of population, exposure, comparator and outcome, PECO). We note that our study will only include non-human focussed SRs as human studies are predominantly correlational, meaning that ancestral environment is not directly manipulated while controlling for other variables, and the outcomes observed in the descendants can be confounded. Occasionally, some human studies may use randomised control trials to assess the effects of pharmaceuticals or medical procedures (e.g., fertility or cancer treatments) on offspring. However, such studies rarely go beyond one generation and the direct effects of pharmaceuticals and medical procedures on offspring are beyond the scope of this map as these manipulations do not fall within our definition of environmental exposures (see "Article screening and study eligibility criteria" section).

Our primary question (question one), followed by three secondary questions (questions two to four), each with a specific rationale, are as follows:

1. What are the broad research patterns unifying the SR literature on non-genetic inheritance across disciplines, such as the most common types of environmental exposures and descendant traits examined? Are there any gaps in the literature? This will provide a clear outline of the current state of the non-genetic inheritance SR literature while clearly highlighting the gaps in the literature that remain to be synthesised, which will streamline the research process by directing researchers to where more research is needed.

2. Are there discipline-specific research patterns including commonalities and disparities between disciplines and what are the patterns of terminology use within and between disciplines? This will highlight how disciplines differ (e.g., do SRs of specific environmental exposures dominate one discipline and not others? Do some disciplines focus on intergenerational effects, and others on trans-generational effects? Do different disciplines use different terminology to describe non-genetic inheritance?) and will allow researchers to not only address research gaps within their own disciplines but also bridge gaps between disciplines.

3. How are authors of the SR literature connected across different countries and disciplines? We will conduct bibliometric analyses of co-author, collaboration, and citation networks. This will highlight interdisciplinary research and main research 'hubs'. This analysis will also reveal to what extent is the cur- rent state of the analysed field is impacted by biases and inequalities existing in research networks.

4. What is the reliability of the SR literature? We will conduct a critical appraisal of the SR literature to assess the rigour, transparency, and risk of bias. This will show the overall reliability of the SR literature, as well as the areas that require improvement.

\section{Methods}

Here, we have adhered to the RepOrting standards for Systematic Evidence Syntheses for systematic map protocols in environmental research (ROSES) [26] (shown in Additional file 1: Appendix S1).

For our Map of Systematic Reviews, we will also report our systematic search and literature selection following (ROSES) [26]. See Fig. 2 for a 'workflow' diagram.

\section{Searching for articles}

We will search three broad-coverage databases-Scopus, ISI Web of Science Core Collection, and PubMed for SRs. The searches will be performed using a custom-designed search string made of three groups of English-language keywords: (1) related to non-genetic inheritance (e.g., maternal, paternal, non-genetic inheritance, inter-generation, trans-generation), (2) related to SR type (e.g., systematic review with or without meta-analysis), and (3) exclusion keywords filtering out most human-centered articles (e.g., men, women, person, worker, patient) as a majority of these studies are purely correlational and we are focussing on SRs that synthesise direct manipulations of the environment (most biomedical studies are experimental studies on rodents). In the search string, we will use database-specific wildcard symbols to include word variants, and Boolean and proximity operators to build efficient and sensitive search strings. We will not use language filters, so we can capture relevant literature published in languages other than English indexed with English-language keywords, titles or abstracts. We will then include studies in languages that we are able to understand (English, Japanese, Polish, Russian). We acknowledge that this may create a language bias, and this will be mentioned in our map of systematic reviews.

We will use the following search string. This search string has been validated through piloting (on Scopus and Web of Science), including literature screening, and a benchmark set of 9 papers (see Additional file 2: Appen$\operatorname{dix}$ S2).

Scopus search string: (TITLE-ABS-KEY ("silver spoon" OR "epigenetic inheritance" OR "non-genetic inheritance" OR "nongenetic inheritance" OR "extended inheritance" OR "extended heritability" OR "developmental programming" OR "developmental" program" OR 


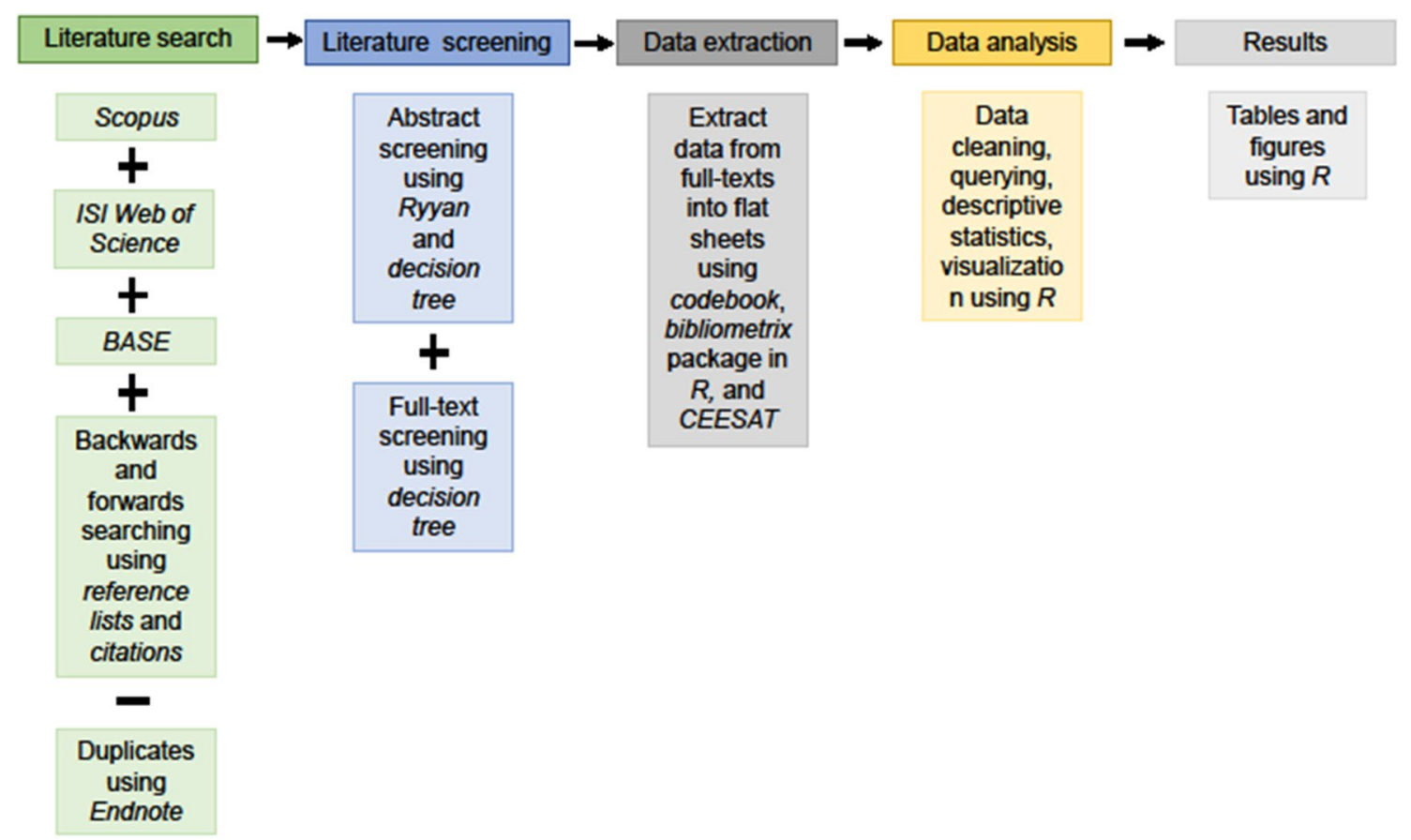

Fig. 2 Workflow diagram for the systematic review map process

"DOHAD" OR "maternal" OR "paternal* OR *paren-


inter-generation* OR across-generation* OR betweengeneration* OR transgeneration* OR multigeneration* OR intergeneration* OR epigenet*) AND (TITLE-ABSKEY (meta-analy* OR metaanaly* OR meta-regress* OR metaregress*) OR TITLE-ABS-KEY ((systematic* OR comprehensiv* OR rapid OR scoping OR quantitativ* OR evidence) W/5 (review* OR map* OR synthes*))) AND NOT TITLE-ABS-KEY (sport* OR econom* OR business* OR software* OR dent* OR orthodont* OR healthcare $^{*}$ OR patient* OR guideline* OR job* OR worker* OR veteran* OR school* OR student" OR child* OR infant* OR baby OR woman OR women OR breast* OR obstetr* OR eclampsia OR family OR placebo OR cancer OR violen* OR hospital* OR diagnos* OR autis* OR educat* OR countries OR china OR africa OR cohort* OR longit* OR rct OR qtl OR gwas OR genome-wide OR age* OR aging OR polyandr* OR chromosom* OR allel* OR genom* OR mutant OR polymorphism OR lifestyle OR leadership OR survey OR comment* OR corrigendum OR erratum)).

Web of Science search string: TS=(("silver spoon" OR "epigenetic inheritance" OR "non-genetic inheritance" OR "nongenetic inheritance" OR "extended inheritance" OR "extended heritability" OR "developmental programming" OR "developmental" program" " OR "DOHAD"
OR *maternal* OR *paternal* OR *parental* OR transgeneration* OR multi-generation* OR inter-generation* OR across-generation* OR between-generation* OR transgeneration* OR multigeneration* OR intergeneration* OR epigenet*) AND ((meta-analy* OR metaanaly* OR meta-regress* OR metaregress* OR evidence) OR (systematic* OR comprehensiv* OR rapid OR scoping OR quantitativ*) NEAR/5 (review* OR map* OR synthes*)) NOT (sport* OR econom* OR business" OR software* OR dent" OR orthodont* OR healthcare* OR patient" OR guideline* OR job* OR worker* OR veteran* OR school* OR student* OR child* OR infant* OR baby OR woman OR women OR breast* OR obstetr* OR eclampsia OR family OR placebo OR cancer OR violen* OR hospital* OR diagnos* OR autis* OR educat* OR countries OR china OR africa OR cohort* OR longit* OR rct OR qtl OR gwas OR genome-wide OR age* OR aging OR polyandr* OR chromosom* OR allel* OR genom* OR mutant OR polymorphism OR lifestyle OR leadership OR survey OR comment* OR corrigendum OR erratum)).

PubMed search string: ("silver spoon"[Title/Abstract]) OR ("epigenetic inheritance"[Title/Abstract]) OR ("non-genetic inheritance"[Title/Abstract]) OR ("nongenetic inheritance"[Title/Abstract]) OR ("extended inheritance"[Title/Abstract]) OR ("extended heritability"[Title/Abstract]) OR ("developmental" 
program*"[Title/Abstract]) OR (DOHAD[Title/Abstract]) OR ("maternal"[Title/Abstract]) OR ("paternal"[Title/ Abstract]) OR ("parental*[Title/Abstract]) OR (transgeneration*[Title/Abstract]) OR (multi-generation*[Title/ Abstract]) OR (inter-generation"[Title/Abstract]) OR (across-generation*[Title/Abstract]) OR (betweengeneration*[Title/Abstract]) OR (transgeneration*[Title/ Abstract]) OR (multigeneration*[Title/Abstract]) OR (intergeneration*[Title/Abstract]) OR (epigenet* [Title/ Abstract]) Filters: Meta-Analysis, Systematic Review, Other Animals.

This search string resulted in 652 hits on Scopus, 816 hits on Web of Science, 280 hits on PubMed and captures all 9 of our preselected benchmark papers (i.e., a validation set used to assess sensitivity of our search and benchmark papers (test set) which are listed in Additional file 2: Appendix S2). Through a pilot literature screening (see Additional file 2: Appendix S2), we estimated that we could get between 50 and 100 relevant full texts for inclusion in our map of SRs, from all planned search sources. Note that we do not include the search term "literature review" as we are not including non-systematic narrative reviews (see "Definition of systematic reviews" below) and the inclusion of this term substantially increases the number of irrelevant search hits in our literature search.

Apart of using direct searches of online literature databases, we will also conduct backwards and forwards searching for additional SRs that may not have been already picked up. These 'snowballing' searches will start from the set of papers included after screening of the above-mentioned online databases. Using Scopus, we will create a custom list of cited and citing papers. We will then pre-filter this list using exact keywords related to review type and screen the remaining references for inclusion in our map, following the same procedure as for the references from the main database searches.

We will also search academic grey literature (Ph.D., Masters and Honours theses) using Bielefeld Academic Search Engine (BASE), with doctype:18* (used for theses) and combinations of English keywords related to nongenetic inheritance (e.g., maternal, paternal, non-genetic inheritance, inter-generation, trans-generation) and keywords related to review type (e.g., systematic, meta-analysis). We will not use language filters in grey literature searches. We will then screen the exported references for inclusion in our review.

We will perform a search update if more than 2 years passes since our original searches were performed before review completion.

\section{Article screening and study eligibility criteria}

We will use Rayyan QCRI [27] for abstract and full text screening. Two independent investigators will each screen all abstracts and full texts following the decision tree shown in Fig. 3. If any discrepancies occur, the two investigators will convene to discuss a logical resolution. If the investigators are unable to agree on a resolution, all authors will convene to decide on a logical resolution. We will record all discrepancies and justifications of the resolutions, and report a screening agreement score.

All search and screening results will be presented in a ROSES-type diagram. We will also provide a list of papers excluded at the full-text stage along with justification for exclusions. We will archive relevant bibliometric files and clearly document any alterations to the predefined protocol.

Language eligibility criteria All included full-text studies must be in English or in a language understood by the review team members (Japanese, Polish, Russian).

Definition of systematic reviews All systematic reviews (SRs) to be included in our map of systematic reviews must be within the 'family' of systematic reviews [23] and must be original or a re-analysis (i.e., providing a new contribution to our understanding of non-genetic inheritance). The SRs will be considered as being part of the SR family if the authors have at least conducted a systematic, rather than ad hoc, literature search and have screened the literature using selection criteria (note that we will conduct a critical appraisal of the included SRs, as mentioned under section "Study validity assessment"). The results of the included SRs may be reported in any form (i.e., a formal or informal metaanalysis, systematic map, rapid review, scoping review, or equivalent). We will exclude reviews not falling into our definition of the systematic review family as these reviews are not considered a rigorous method for synthesising the literature and cannot be critically assessed for quality with the established tools like CEESAT.

We will determine if a publication is eligible for inclusion in our map of systematic reviews by assessing if it meets our selection criteria specified using the PECO (Populations, Exposure, Comparators, Outcomes; [28] framework, as below).

Population We will include SRs on non-human species where there is clear separation of generations, as in most animals and plants. We will exclude SRs that focus exclusively on taxa that use asexual reproduction without clear separation of generations, such as budding, fragmentation or vegetative propagation. All included SRs must use experimental primary literature and must not solely use correlational studies due to the inability to directly assess the effects of environmental factors through manipulation while controlling for other factors in correlational studies. SRs exclusively on humans are excluded from our review as most human 


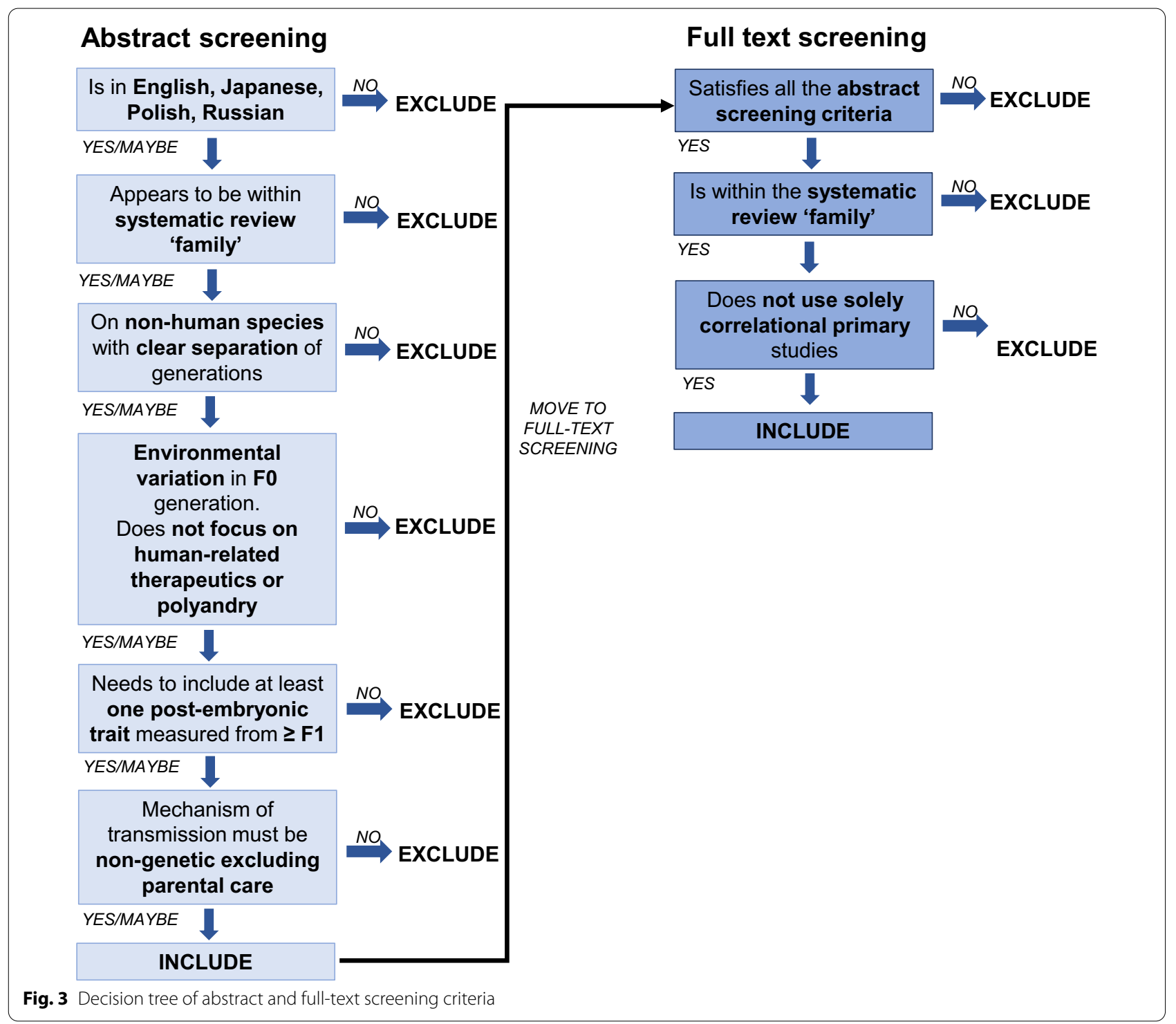

studies are purely correlational. Instead, we will include biomedical studies conducted on animals with goal of being applicable to humans, if they fulfil our inclusion criteria.

Exposure We will include SRs of F0 (ancestral generation) environmental exposures that fall within the following broad categories: (1) diet, (2) human-induced environmental pollutants/toxins, (3) natural variation in environmental composition (e.g., salt, nitrogen), (4) psychological stress (e.g., post-natal separation), (5) temperature, (6) 'human health risk' related environmental exposures (e.g., tobacco, alcohol), (7) differences in population demographics (e.g., population density, sex-ratio, impact of predators/grazers), (8) light and/or photoperiod, (9) other.
We will not include SRs that focus exclusively on human-related therapeutics/pharmaceutical drugs, such as medicines and physical procedures like surgeries and injections, as these are direct manipulations of the individual rather than a manipulation of the environment. However, we will include the effects of human-related medicines if such SRs are related to environmental toxicology and are investigating the effects of medicines as environmental pollutants. We will also not include any SRs that exclusively focus on the effects of polyandry (i.e., how many males the F0 female mates with) because differences in offspring phenotype are likely to be due to differences in paternity as females of some species can have mixed-paternity broods or bias sperm use from certain males [29]. 
The mode of trans-generational transmission considered in the SR must be non-genetic, with gametic or embryonic mode of transmission. We will record if the SR includes studies of factors that may induce DNA mutations. We will not include syntheses where the effects of F0 environment are conferred to offspring exclusively through parental care as this mechanism of the non-genetic inheritance occurs postnatally/posthatching rather than through gametic or embryonic nongenetic transmission.

Comparators SRs need to compare F0 environmental exposure from within the categories presented above to either a control environment (e.g., standard laboratory or natural conditions deemed optimal for a given species) or two different 'levels' of the same type of environment (i.e., hot vs. cold temperature, high nutrient concentration vs low nutrient concentration).

Outcomes SRs must focus on the effects of F0 environment on phenotypic traits that are measured in F1 and/ or subsequent (F2, F3...) generations. SRs will be categorised as including inter- and/or trans-generational effects (or mixture thereof), based on the focal taxa and the F0 sex exposed (see Fig. 1).

We will exclude SRs that entirely focus on F0 female fecundity or fertility (i.e., the number of offspring) as these are considered maternal traits rather than descendants' traits. Descendants' traits will be classified into the following broad categories: (1) physiological (e.g., immune function, insulin levels, hormone levels), (2) morphological (e.g., body size, adiposity, colouration), (3) reproductive (e.g., fecundity measures), (4) life-history (e.g., developmental rate, lifespan and aging), (5) behavioural (e.g., response to stimuli, anxiety, learning), (6) molecular (e.g., gene expression, DNA methylation), (7) health and disease (e.g., disease prevalence), and (8) other [30].

\section{Study validity assessment}

We will conduct a critical appraisal of the quality of the SRs (i.e., rigour and transparency of methods, and risk of bias) following the Collaboration for Environmental Evidence Synthesis Assessment Tool (CEESAT) Version 2.1 criteria (Additional file 5: Appendix S5; https:// environmentalevidence.shinyapps.io/CEEDER/). We will ensure that any reviewers participating in this map of SRs do not critically appraise any of their own SRs. This will be avoided by delegating the critical appraisal task to a reviewer that is not an author on the SR being assessed. Authors will not be able to provide any advice or input to the critical appraisal of their SR. The validity of critical appraisal assessments will be cross-checked by a second reviewer through independent assessment.

\section{Data coding strategy}

For each SR that meets our abstract and full text screening criteria, we will extract details pertaining to three broad categories (see Additional file 3: Appendix S3 for a full codebook of data to be extracted and its coding; Fig. 4 for which data elements will be used to address which objective; https://osf.io/detvk/ for pilot data extraction and codebook of extracted data elements; Additional file 4: Appendix S4 for a relational diagram of how all flat sheets and data elements in Additional file 3: Appendix S3 are connected).

1. Bibliometric information, such as publication title, year, journal, authors and affiliations, broad research discipline, and key words used to describe the paper. Note that some bibliometric data for the bibliometric analysis (i.e., keywords, co-authors and affiliations) will not be extracted manually using the codebook, but will be extracted directly from Scopus records using the bibliometrix package [31] in $R$ [32].

2. Information regarding the F0 environmental exposures, such as the types of environmental factors, whether the environmental exposures were predicted by the authors to have positive, negative, or neutral effect on descendants, what life stage the F0 generation was exposed to the environmental factor (i.e., as a juvenile/prior to sexual maturity or as an adult), and what sex was exposed to the environment (i.e., are non-genetic effects conferred through the matriline, patriline, or both).

3. Information regarding the outcomes on the descendants, such as if the authors claimed non-genetic effects are inter- or trans-generational (assessed based on the filial generation measured, and taxa; see Fig. 1-we will record if the authors terminology use matches our definition of inter- and trans-generational), the generation of the descendants (i.e., F1, F2, F3... etc.), the traits measured, and at what life stage the traits were measured.

Data will be extracted to pre-piloted flat sheets in Excel with dropdown lists of categories to limit errors (see https://osf.io/detvk/ for pilot raw data). Each SR will be coded with an identifiable unique study ID that will be used to connect relevant data from each SR between different flat sheets when imported into the statistical analysis program $R$ [32]. Note that data extraction for some SRs will involve 'plural' data elements whereby the details of the SR fall into multiple categories. For example, the SR might cover multiple environmental categories, trait categories, or involve both inter- and trans-generational effects. Such SRs will be coded accordingly by assigning multiple levels of the plural elements. Each plural variable 


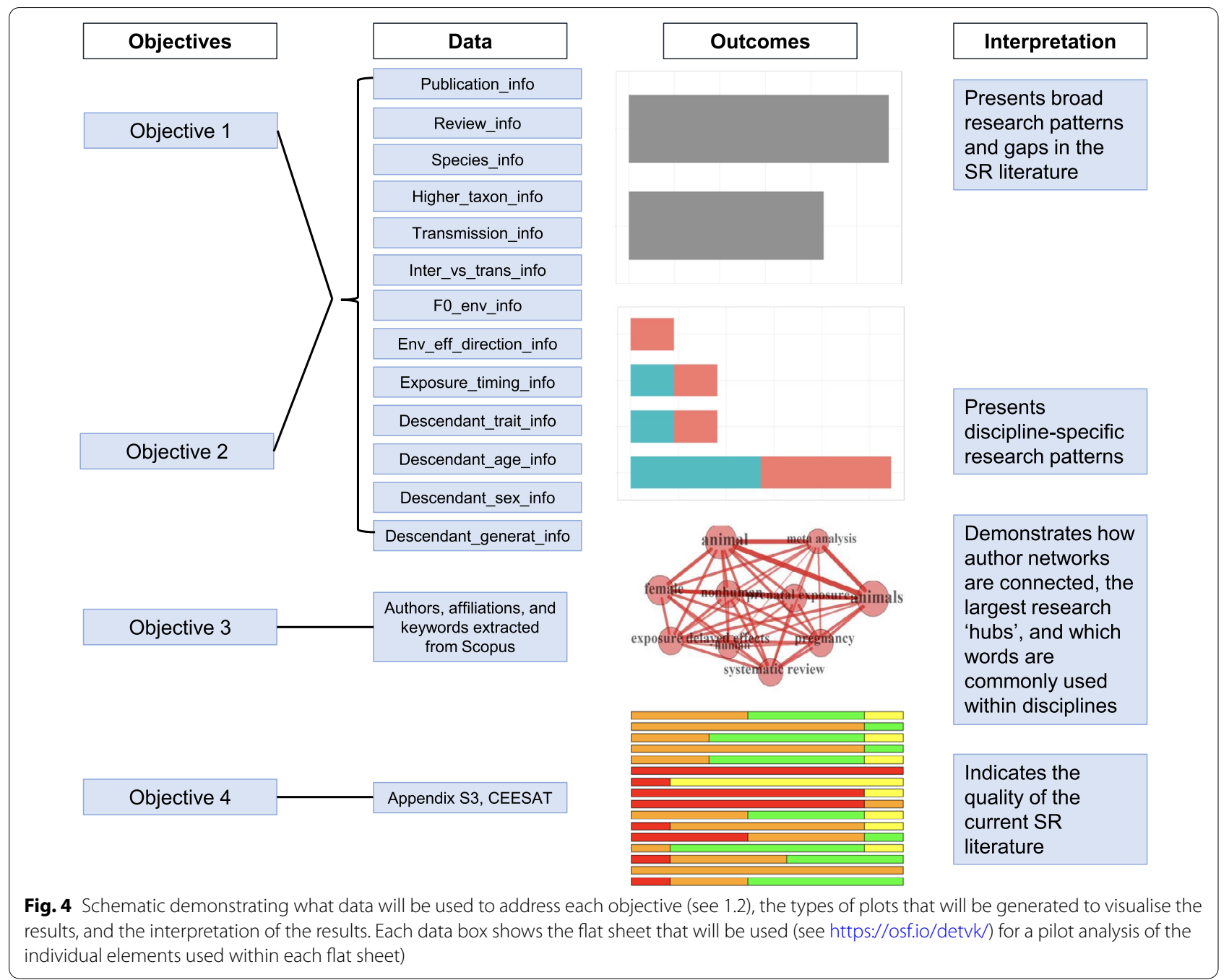

will be recorded on a separate flat sheet (see Additional file 3: Appendix S3 for an indication of which variables are predicted to be plural or singular and https://osf.io/ detvk/ for pilot data).

A single investigator will extract the data following the codebook (Additional file 3: Appendix S3). A second investigator will cross-check the extracted data from $20 \%$ of the relevant SRs. If any discrepancies occur, the two investigators will convene to discuss a logical resolution. If the investigators are unable to agree on a resolution, all authors will convene to decide on a logical resolution. All discrepancies and justifications of the resolution will be recorded, and if the percentage of disagreements for a given variable exceeds $5 \%$, all extracted records of that variable will be further cross-checked by the second reviewer [30]. We note that our pilot data collection and cross-checking resulted in $98 \%$ consistent results.

It must also be noted that we expect some of the broader SRs (particularly in ecology) to include some primary studies that do not meet our inclusion criteria (i.e., SRs might include a mixture of both experimental and observational primary studies). These SRs will still be included and plurally coded with the different factor levels, hence why some of the factor levels used in the codebook are in our exclusion criteria but will still be coded in the data.

All extracted data and meta-data will be open access and published in the Open Science Framework (https:// osf.io/detvk/).

\section{Study mapping and presentation}

The data collected above will allow us to address our objectives. Figure 4 shows which data elements will be queried, how results will be presented and the interpretation of these outcomes. See https://osf.io/detvk/ for pilot data synthesis.

During initial processing of extracted raw data, we will load each flat sheet into the statistical program, 
$R$ [32]. We then will join these sheets using the corresponding study IDs. vocabulary. We will then check the data for errors in data entry and wrangling.

We will be able to address each objective by querying the corresponding data elements and presenting the results in easily interpretable outcomes such as counts, distributions and descriptive statistics (Fig. 4). These results will be presented in tables and figures, such as bar graphs and heatmaps (for mapping data, including knowledge gaps and clusters; created using ggplot 2 $\mathrm{R}$ package [33]), networks (for bibliometric analyses, e.g., collaboration network, term-co-occurrence network, created using bibliometrix and igraph $\mathrm{r}$ packages [31, 34]) and traffic light plots (for critical assessment using CEESAT). As an example, we present preliminary analyses of the pilot data extractions in a downloadable interactive file Rmarkdown-pilot.html, available from https://osf.io/detvk/ (we note that the styling and arrangement of tables and plots may be different in the resulting manuscript).

Finally, we will narratively discuss the results and interpretations pertaining to each objective (see Fig. 4 for a description of the interpretation of each objective). This will be written under appropriate headings and subheadings to aid readability. All limitations of our approach and data will be acknowledged and discussed.

\section{Supplementary Information}

The online version contains supplementary material available at https://doi. org/10.1186/s13750-021-00245-9.

Additional file 1: Appendix S1. ROSES checklist. Completed ROSES checklist.

Additional file 2: Appendix S2. Pilot search string and benchmark papers. Search string used for pilot literature screening and validating against benchmark papers.

Additional file 3: Appendix S3. Full Code Book. Codebook documenting all data elements and levels to be extracted.

Additional file 4: Appendix S4. Relational Diagram. Relational diagram showing how all data elements are connected.

Additional file 5: Appendix S5. CEESAT Critical Appraisal tool. Checklist used for assessing included reviews.

\section{Acknowledgements}

We acknowledge our colleagues at the Evolution \& Ecology Research Centre and the School of Biological, Earth and Environmental Sciences, and the Faculty of Science at the University of New South Wales for the moral and infrastructural support.

\section{Authors' contributions}

SN and ML conceived the idea and attained funding. ELM and ML conducted piloting. ELM drafted the protocol. SMD, SN, ML reviewed and edited the protocol. All authors read and approved the final manuscript.

\section{Funding}

This project was funded by an Australian Research Council Discovery Project Grant awarded to SN and ML (DP200100367).

\section{Data availability}

All extracted data will be hosted in a dedicated GitHub repository and also archived as an OSF project. Extracted data will be stored as flat .csv files, long format, with an associated meta-data file defining columns and responses as a text file. The datasets generated and/or analysed during the current study are available in the Open Science Framework, https://osf.io/detvk/. All data will be freely downloadable upon publication of the resulting manuscript.

\section{Code availability}

All extracted code will be hosted in a dedicated GitHub repository and also archived as an OSF project. The code will be stored in .R or .Rmd file format. All code will be freely downloadable upon publication of the resulting manuscript.

\section{Declarations}

Ethics approval and consent to participate

Not applicable.

\section{Consent for publication \\ Not applicable.}

\section{Competing interests}

The authors have no competing interests to declare.

Received: 25 August 2021 Accepted: 22 October 2021

Published online: 06 November 2021

\section{References}

1. Veenendaal MVE, Painter RC, De Rooij SR, Bossuyt PMM, Van Der Post JAM, Gluckman PD, et al. Transgenerational effects of prenatal exposure to the 1944-45 Dutch famine. BJOG Int J Obstet Gynaecol. 2013;120:548-54.

2. Donelson JM, Munday PL, Mccormick MI, Pitcher CR. Rapid transgenerational acclimation of a tropical reef fish to climate change. Nat Clim Change. 2012;2:2011-3.

3. Mousseau TA, Fox CW. The adaptive significance of maternal effects. Trends Ecol Evol. 1998:13:403-7.

4. Carone BR, Fauquier L, Habib N, Shea JM, Hart CE, Li R, et al. Paternally induced transgenerational environmental reprogramming of metabolic gene expression in mammals. Cell. 2010;143:1084-96.

5. Lambrot R, Xu C, Saint-Phar S, Chountalos G, Cohen T, Paquet M, et al. Low paternal dietary folate alters the mouse sperm epigenome and is associated with negative pregnancy outcomes. Nat Commun. 2013:4:1-13.

6. Waterland RA, Jirtle RL. Transposable elements: targets for early nutritional effects on epigenetic gene regulation. Molecular and cellular biology. Am Soc Microbiol. 2003;23:5293-300.

7. Saavedra-rodríguez L, Feig LA. Defective social interactions across generations. Biol Psychiatry. 2013;73:44-53.

8. Kubsad D, Nilsson EE, King SE, Sadler-riggleman I, Beck D, Skinner MK. Assessment of glyphosate induced epigenetic transgenerational inheritance of pathologies and sperm epimutations: generational toxicology. Sci Rep. 2019;9:1-17.

9. Wylde Z, Spagopoulou F, Hooper AK, Maklakov AA, Bonduriansky R. Parental breeding age effects on descendants' longevity interact over 2 generations in matrilines and patrilines. PLoS Biol. 2019;17:1-23.

10. Kremsky I, Corces VG. Protection from DNA re-methylation by transcription factors in primordial germ cells and pre-implantation embryos can explain trans-generational epigenetic inheritance. Genome Biol. 2020;21:1-31.

11. Martin C, Zhang Y. Mechanisms of epigenetic inheritance. Curr Opin Cell Biol. 2007;19:266-72.

12. Messerschmidt DM. Should I stay or should I go, protection and maintenance of DNA methylation at imprinted genes. Epigenetics. 2012;7:969-75. 
13. Adrian-Kalchhauser I, Sultan SE, Shama LNS, Spence-Jones H, Tiso S, Keller Valsecchi Cl, et al. Understanding "non-genetic" inheritance: insights from molecular-evolutionary crosstalk. Trends Ecol Evol. 2020;35:1078-89.

14. Jirtle RL, Skinner MK. Environmental epigenomics and disease susceptibility. Nat Rev Genet. 2007:8:253-62.

15. Shukla A, Bunkar N, Kumar R, Bhargava A, Tiwari R, Chaudhury K, et al. Air pollution associated epigenetic modifications: transgenerational inheritance and underlying molecular mechanisms. Sci Total Environ. 2019:656:760-77.

16. Jablonka EVA, Raz GAL. Transgenerational epigenetic inheritance: prevalence, mechanisms, and implications for the study of heredity and evolution. Q Rev Biol. 2009;84:131-76.

17. Burgueño AL, Juárez YR, Genaro AM, Tellechea ML. Prenatal stress and later metabolic consequences: systematic review and meta-analysis in rodents. Psychoneuroendocrinology. 2020;113:1-8.

18. Hodjat M, Rezvanfar MA, Abdollahi M. A systematic review on the role of environmental toxicants in stem cells aging. Food Chem Toxicol. 2015;86:298-308.

19. Caixeta MB, Araújo PS, Gonçalves BB, Silva LD, Grano-Maldonado MI, Rocha TL. Toxicity of engineered nanomaterials to aquatic and land snails: a scientometric and systematic review. Chemosphere. 2020;260:127654.

20. Uller T, Nakagawa S, English S. Weak evidence for anticipatory parental effects in plants and animals. J Evol Biol. 2013;26:2161-70.

21. Yin J, Zhou M, Lin Z, Li QQ, Zhang YY. Transgenerational effects benefit offspring across diverse environments: a meta-analysis in plants and animals. Ecol Lett. 2019;22:1976-86.

22. James KL, Randall NP, Haddaway NR. A methodology for systematic mapping in environmental sciences. Environ Evid. 2016;5:1-13.

23. Moher D, Stewart L, Shekelle P. All in the family: systematic reviews, rapid reviews, scoping reviews, realist reviews, and more. Syst Rev. 2015:4(1):1-2
24. Woodcock P, Pullin AS, Kaiser MJ. Evaluating and improving the reliability of evidence syntheses in conservation and environmental science: a methodology. Biol Conserv. 2014;176:54-62

25. Nakagawa S, Samarasinghe G, Haddaway NR, Westgate MJ, O'Dea RE, Noble DWA, et al. Research weaving: visualizing the future of research synthesis. Trends Ecol Evol. 2019;34:224-38.

26. Pussegoda K, Turner L, Garritty C, Mayhew A, Skidmore B, Stevens A, et al. Systematic review adherence to methodological or reporting quality. Syst Rev. 2017:6:1-14.

27. Ouzzani M, Hammady H, Fedorowicz Z, Elmagarmid A. Rayyan-a web and mobile app for systematic reviews. Syst Rev. 2016;5:1-10.

28. Koricheva J, Gurevitch J, Mengersen K. Handbook of meta-analysis in ecology and evolution. Princeton: Princeton University Press; 2013.

29. Eberhard WG, Cordero C. Sexual selection by cryptic female choice on male seminal products - a new bridge between sexual selection and reproductive physiology. Trends Ecol Evol. 1995;10:493-6.

30. Bland M. An introduction to medical statistics. Oxford: Oxford University Press; 2015

31. Aria M, Cuccurullo C. Bibliometrix: an R tool for comprehensive analysis of scientific literature. J Informet. 2017;11:959-75.

32. R Core Team. R: a language and environment for statistical computing. Vienna, Austria; 2020. http://www.r-project.org/.

33. Wickham H. ggplot2: elegant graphics for data analysis. New York: Springer; 2016. https://ggplot2.tidyverse.org.

34. Csardi G, Nepusz T. The igraph software package for complex network research. InterJournal. 2006. https://igraph.org.

\section{Publisher's Note}

Springer Nature remains neutral with regard to jurisdictional claims in published maps and institutional affiliations.
Ready to submit your research? Choose BMC and benefit from:

- fast, convenient online submission

- thorough peer review by experienced researchers in your field

- rapid publication on acceptance

- support for research data, including large and complex data types

- gold Open Access which fosters wider collaboration and increased citations

- maximum visibility for your research: over $100 \mathrm{M}$ website views per year

At BMC, research is always in progress.

Learn more biomedcentral.com/submissions 